and fasting duration. Log transformation was used for TG and insulin levels.

Results Independently of current SEP, lower childhood SEP was significantly associated with higher $\operatorname{logTG}(\beta=0.013, p=0.042)$, higher $\mathrm{LDL}(\beta=1.979, p=0.023)$, higher body fat percentage $(\beta=0.476$, $p=0.025)$ and higher odds of physical inactivity $(O R=1.13, p=0.043)$. Independently of childhood SEP, lower adulthood SEP was significantly associated with lower HDL level $(\beta=-0.934, p=0.026)$, higher WHR ( $\beta=0.006, p=0.002)$, and higher odds of smoking ( $O R=1.31$, $\mathrm{p}=0.001)$ and physical inactivity $(\mathrm{OR}=1.34, \mathrm{p}<0.0001)$. Significant interactions were found between SEP in both time points and LDL, WHR and WC ( $p$ for interactions $=0.01,0.024$ and 0.055 respectively). The detrimental effect of lower SEP in adulthood on LDL level was strongest among subjects with high SEP in childhood $(\beta=4.54, p<0.0001)$, compared with those having lower SEP in childhood. Similar trends were observed for WHR and WC $(\beta=0.011$, $p<0.0001$ and $\beta=1.754, p=0.004$ for associations of adulthood SEP with WHR and WC respectively, among subjects with high childhood SEP). Analyses, based on education as a measure of SEP, yielded similar interactive patterns.

Conclusion Adverse SEP at both childhood and adulthood has an independent influence on physiological and behavioral risk factors. The interaction between SEP in childhood and adulthood in their effect on cardiometabolic risk factors points to the important role played by social mobility in affecting adult poorer health.

\section{OP11 THE ASSOCIATION OF CHILDHOOD ADIPOSITY, AND ITS DISTRIBUTION, WITH CARDIOMETABOLIC OUTCOMES: RESULTS FROM 13 EUROPEAN COHORTS}

doi:10.1136/jech-2012-201753.011

S Patel, DA Lawlor, CHICOS collaborative project. MRC Centre for Causal Analyses in Translational Epidemiology, School of Socal and Community Medicine, University of Bristol, Bristol, UK

Background Adiposity in childhood has been associated with increased cardiovascular risk which may be established in childhood and adolescence. Also centrally distributed fat (waist circumference) has been suggested to be a better marker of cardiometabolic risk compared to body mass index (BMI). We aimed to assess the association of adiposity measures with cardiometabolic outcomes in children and consider if the magnitude of these associations differ by adiposity measurement.

Methods European cohorts with data available on BMI and waist circumference and cardiometabolic outcomes (at least blood pressure) were invited to participate in the collaborative project. Crosssectional associations between adiposity measures (age- and gender-standardised $\mathrm{z}$ scores) and cardiometabolic outcomes and prospective associations (cardiometabolic outcomes measured at least 12 months after adiposity measurement) were examined. Results from individual cohorts were pooled using a random-effect meta-analysis and heterogeneity between them explored.

Results A total of 13 cohorts ( $n=3644-17186$ for different outcomes) were included in the cross-sectional analysis. BMI and waist circumference were both positively associated with diastolic and systolic blood pressure (DBP, SBP) and with total cholesterol, LDLc, triglycerides, insulin and CRP, with the magnitudes of association with each outcome being similar for BMI and waist. For example, the mean difference in DBP per standard deviation (SD) increase in BMI was $0.90 \mathrm{mmHg}(95 \%$ confidence intervals (CI) $0.55,1.24)$ and per SD waist was $0.73 \mathrm{mmHg}(95 \% \mathrm{CI} 0.33,1.12)$; similar results for SBP were $1.73 \mathrm{mmHg}(95 \% \mathrm{CI} 1.28,2.18)$ and $1.33 \mathrm{mmHg}(95 \% \mathrm{CI}$ $0.83,1.81$ ). In prospective analysis ( 6 cohorts, $n=3708-7073$ for different outcomes) BMI and waist circumference were positively associated with SBP and total cholesterol and inversely with HDLc associations were similar for the two adiposity measurements or were stronger for BMI. For example a $1 \mathrm{SD}$ greater BMI was associated with $1.36 \mathrm{mmHg}$ higher SBP (95\%CI 0.67, 2.05) compared with $0.96 \mathrm{mmHg}(95 \%$ CI $0.35,1.57)$ for waist. Direct measurements of fat mass, such as DXA or bioelectrical impedance, did not exhibit stronger associations with risk factors than did BMI. Age at adiposity measurement did not consistently influence the heterogeneity of association between studies.

Conclusion Adiposity in childhood and adolescence is associated with adverse cardiometabolic outcomes. This suggests that interventions should be considered earlier before adverse effects become established. The magnitude of the association is similar between BMI, waist circumference and direct measures of adipostiy. Therefore BMI alone could be considered an adequate measure in public health and health surveillance systems.

\section{OP12 CHILDHOOD INFECTIOUS DISEASE AND RISK OF PREMATURE DEATH FROM CANCER: A PROSPECTIVE COHORT STUDY}

doi:10.1136/jech-2012-201753.012

1PWG Tennant, ${ }^{2} \mathrm{~L}$ Parker, ${ }^{3} \mathrm{JE}$ Thomas, ${ }^{4} \mathrm{AW}$ Craft, ${ }^{1} \mathrm{MS}$ Pearce. ${ }^{1}$ Institute of Health \& Society, Newcastle University, Newcastle upon Tyne, UK; ${ }^{2}$ Departments of Medicine and Pediatrics, Population Cancer Research Program, Halifax, Canada; ${ }^{3 R o y a l}$ Victoria Infirmary, Newcastle upon Tyne Hospitals NHS Foundation Trust, Newcastle upon Tyne, UK; ${ }^{4}$ Northern Institute of Cancer Research, Newcastle University, Newcastle upon Tyne, UK

Background The hygiene-hypothesis proposes that early-life infectious diseases may protect against a number of adverse health conditions later in life, most notably the development of cancer. Existing investigations of this association, however, have provided equivocal results, possibly due to inadequate adjustment for confounding or the use of retrospective exposure information. This study utilised longitudinal data from the Newcastle Thousand Families Study, a prospective cohort of 1147 individuals born in Newcastle-upon-Tyne (UK) in 1947, to assess the impact of various childhood infectious diseases on death from cancer between ages 15 and 60 years.

Methods Detailed information was collected prospectively at birth and during childhood on a number of early life factors. Episodes of illness during the first 15 years were obtained routinely by health visitors, who regularly visited the participants' homes, or directly from the family doctor or hospital. All study members were 'flagged' by the UK National Health Service Central Register when they died or emigrated. Deaths from cancer between ages 15 to 60 years were analysed in relation to childhood infections, adjusting for potential early-life confounders, using Cox proportional-hazards regression.

Results Of the 1060 study members known to be alive at age 15 years, 88 died before aged 60 years, including 37 from cancer - the leading cause of death. Childhood history of measles and childhood history of influenza, were both independently associated with a lower risk of death from cancer during ages 15 to 60 years (influenza: adjusted hazard ratio, $\mathrm{aHR}=0.39,95 \% \mathrm{CI}$ : 0.17-0.88, $\mathrm{p}=0.03$; measles: $\mathrm{aHR}=0.49,95 \% \mathrm{CI}: 0.24-0.98, \mathrm{p}=0.04)$. In contrast, childhood history of whooping cough was associated with a higher risk of death from cancer during ages 15 to 60 years (adjusted $\mathrm{HR}$, $\mathrm{aHR}=4.88$, 95\% CI: 2.29-10.39, p<0.0001). The association between whooping cough and cancer-related mortality was borderline significantly different between men and women $(p=0.05)$, with a stronger association among women than men (women: $a \mathrm{HR}=12.20,95 \% \mathrm{CI}$ : 3.01-49.42, $p=0.0005$; men: $a H R=2.10,95 \%$ CI: 0.80-5.50, $p=0.13$ ).

Conclusion In a pre-vaccination cohort from the North of England, childhood infection with measles and influenza were associated with a reduced risk of death from cancer in adulthood, while childhood whooping cough was associated with an increased risk. These results suggest that there may be some disease-specific associations between childhood infectious diseases and death from adult cancer, however further studies are required to confirm the specific associations identified. 